\title{
Melanoma Metastases to the Adrenal Gland Are Highly Resistant to Immune Checkpoint Inhibitors
}

\author{
Jessica S.W. Borgers, MD ${ }^{1,2, *}$; Richard P. Tobin, $\mathrm{PhD}^{1,3,4, *}$; Robert J. Torphy, $\mathrm{MD}^{1}$; \\ Victoria M. Vorwald, BS ${ }^{1,3,4}$; Robert J. Van Gulick, $\mathrm{BS}^{3,4,5}$; Carol M. Amato, MS ${ }^{3,4,5}$; \\ Dasha T. Cogswell, MS ${ }^{1,3,4}$; Tugs-Saikhan Chimed, MS ${ }^{5}$; Kasey L. Couts, $\mathrm{PhD}^{4,5}$; Adrie Van Bokhoven, $\mathrm{PhD}^{6}$; \\ Christopher D. Raeburn, MD; Karl D. Lewis, MD ${ }^{4,5}$; Joshua Wisell, MD ${ }^{4,6}$; Martin D. McCarter, MD ${ }^{1,3,4}$; \\ Rao R. Mushtaq, $\mathrm{MD}^{5}$; and William A. Robinson, $\mathrm{MD}, \mathrm{PhD}^{3,4,5}$
}

\begin{abstract}
Background: Adrenal gland metastases (AGMs) are common in advanced-stage melanoma, occurring in up to $50 \%$ of patients. The introduction of immune checkpoint inhibitors (ICls) has markedly altered the outcome of patients with melanoma. However, despite significant successes, anecdotal evidence has suggested that treatment responses in AGMs are significantly lower than in other metastatic sites. We sought to investigate whether having an AGM is associated with altered outcomes and whether $\mathrm{ICl}$ responses are dampened in the adrenal glands. Patients and Methods: We retrospectively compared $\mathrm{ICl}$ responses and overall survival (OS) in 68 patients with melanoma who were diagnosed with an AGM and a control group of 100 patients without AGMs at a single institution. Response was determined using RECIST 1.1. OS was calculated from time of $\mathrm{ICl}$ initiation, anti-PD-1 initiation, initial melanoma diagnosis, and stage IV disease diagnosis. Tumor-infiltrating immune cells were characterized in 9 resected AGMs using immunohistochemical analysis. Results: Response rates of AGMs were significantly lower compared with other metastatic sites in patients with AGMs (16\% vs $22 \%)$ and compared with those without AGMs (55\%). Patients with AGMs also had significantly lower median OS compared with those without AGMs (3.1 years vs not reached, respectively). We further observed that despite this, AGMs exhibited high levels of tumor-infiltrating immune cells. Conclusions: In this cohort of patients with melanoma, those diagnosed with an AGM had lower ICl response rates and OS. These results suggest that tissue-specific microenvironments of AGMs present unique challenges that may require novel, adrenal gland-directed therapies or surgical resection.
\end{abstract}

J Natl Compr Canc Netw, doi: 10.6004/jnccn.2020.7800 Published online August 4, 2021

\footnotetext{
${ }^{1}$ Division of Surgical Oncology, Department of Surgery, University of Colorado Anschutz Medical Campus, Aurora, Colorado; ${ }^{2}$ The Netherlands Cancer Institute, Amsterdam, the Netherlands; and ${ }^{3}$ Center for Rare Melanomas, ${ }^{4}$ International Melanoma Biorepository, Center for Rare Melanomas, ${ }^{5}$ Division of Medical Oncology, Department of Medicine, ${ }^{6}$ Department of Pathology, and

${ }^{7}$ Division of GI, Trauma, and Endocrine Surgery, Department of Surgery,

University of Colorado Anschutz Medical Campus, Aurora, Colorado.
}

\section{Background}

Despite significant advances in the understanding of the etiology and treatment of melanoma, its incidence continues to increase and 5-year survival rates for advanced stages remain low. ${ }^{1}$ Major breakthroughs such as the discovery of targeted therapies and immunotherapies, including the immune checkpoint inhibitors (ICIs) targeting CTLA-4, PD-1, and PD-L1, have improved survival rates for many patients with cancer, and in particular those with melanoma. ${ }^{2-4}$ Because of the increasing number of patients treated with these therapies, there is now an ever-expanding patient population with intrinsic or acquired immunotherapy resistance with few therapeutic options beyond first-line treatments.

In advanced-stage melanoma, clinical trials have shown objective response rates of approximately $40 \%$ and a reduction in overall tumor burden in $60 \%$ to $70 \%$ of patients treated with anti-PD-1 therapies. ${ }^{5-7}$ Furthermore, several studies have shown that there are differences in response rates according to the size and location of the metastases, favoring the responses of small lesions and lung metastases. ${ }^{8-10}$ Interestingly, liver metastases show a differential response based on the extent of CD8 + T-cell tumor infiltration, as well as T-cell PD-1 and tumor PD-L1 expression. Taken together, these findings suggest that the tissue-specific tumor microenvironment differs between various metastatic sites and between patients. ${ }^{8-10}$ In line with these findings, Nguyen et $\mathrm{al}^{11}$ reported a case series of 3 patients with progression of adrenal gland metastases (AGMs) despite objective responses to the anti-PD-1 therapy pembrolizumab in all other metastatic sites. This supports the hypothesis that certain tissues in the body may possess tissuespecific resistance mechanisms because of different tumor microenvironments.

See JNCCN.org for supplemental online content. 
Currently, there are limited data reporting specific response rates of AGMs to immunotherapy in patients with metastatic malignant melanoma. Postmortem examination of patients with advanced-stage melanoma has found metastases to the adrenal gland in approximately $50 \%$ of cases. ${ }^{12}$ This makes the adrenal gland the fourth most common site of metastasis after lung, liver, and bone. ${ }^{13}$ Due to their key role in the stress response system, the adrenal glands are among the most highly vascularized tissues in the human body. ${ }^{14,15}$ Although the exact mechanism resulting in preferential metastasis to the adrenal gland is unknown, it has been suggested that the rich supply of sinusoidal blood may provide a potential explanation. ${ }^{13,16}$ Adrenal insufficiency caused by metastatic disease is rare, as a significant portion of both adrenal glands must be affected to be clinically apparent. ${ }^{13,17}$ Due to the lack of clinical symptoms and biochemical changes, diagnosis of adrenal gland involvement is often an incidental finding. ${ }^{18}$

In addition to the medulla's role in the stress response, the adrenal cortex plays an important role in the regulation of inflammatory responses through the production of immunologically active molecules, including corticosteroids. ${ }^{19}$ Accordingly, systemic administration of glucocorticoids has both anti-inflammatory and immunosuppressive effects, mainly caused by alterations in the distribution and function of lymphocytes, monocytes, and neutrophils. ${ }^{20,21}$ Glucocorticoids have also been shown to inhibit T-cell activation, leading to decreased proliferation and cytokine production. ${ }^{20,21}$ However, because immunotherapies are designed to promote immune activation, systemic or local glucocorticoids may induce immunosuppression and inhibit the efficacy of these therapies. As such, the increased tissue-specific concentrations of glucocorticoids within the adrenal glands may provide one of several mechanisms of immune escape for metastatic melanoma. Therefore, we hypothesized that the adrenal glands are distinguished from other sites of melanoma metastases by the hostility of their microenvironments to antitumor immune cells, making the adrenal glands highly permissive to melanoma metastases and limiting the efficacy of ICIs.

\section{Patients and Methods}

\section{Patient Selection and Information}

This single-center, retrospective study included patients with stage IV melanoma aged $\geq 18$ years who received at least 1 dose of an ICI and had sufficient follow-up information to perform our analyses at the UCHealth University of Colorado Hospital (UCH) between 2008 and 2018. A total of 68 patients with unilateral or bilateral AGMs and 100 patients with stage IV disease without AGMs were identified. Patients without a valid research consent, incomplete medical records, or inadequate follow-up information were excluded. The following patient and tumor characteristics were collected from electronic medical records: sex, age at melanoma diagnosis, followup in years, overall survival (OS), subtype of primary melanoma, stage at diagnosis, mutation status, metastatic sites, and treatments received. In the group of patients with an AGM, the time to AGM and side of AGM was obtained, as well as the date of surgery for patients who underwent an adrenalectomy. Patients were well matched for age at diagnosis, age at initial treatment, sex, and therapies received (Table 1).

Immunotherapies were defined as antibodies targeting the immune checkpoints CTLA-4 (ipilimumab), PD-1 (nivolumab, pembrolizumab), and PD-L1 (atezolizumab), together referred to as ICIs, and drugs to improve the antitumor immune response (eg, IL-2). Molecular targeted therapies were defined as treatments designed to target specific molecules involved in the growth and spread of cancer cells, and included dabrafenib, trametinib, cobimetinib, vemurafenib, regorafenib, insulin-like growth factor 1 (IGFR-1) inhibitors, apomab, and PI-88. Chemotherapies were defined as agents designed to kill tumor cells directly, without targeting specific molecules, and included cisplatin, vinblastine, dacarbazine, carmustine, paclitaxel, capecitabine, temozolomide, and vincristine sulfate liposome.

Written informed consent was obtained from all patients according to Colorado Multiple Institutional Review Board Protocol 05-0309.

\section{Response}

To determine individual response, the best response to ICIs was determined by a trained radiologist according to RECIST 1.1 imaging criteria. In the group of patients who underwent an adrenalectomy, the best response prior to surgical removal was used.

\section{Pathology}

Nine patients who underwent a unilateral or bilateral adrenalectomy were identified. Tissue slides were cut from formalin-fixed paraffin-embedded blocks. Slides were then deparaffinized and rehydrated in graded concentrations of alcohol using standard techniques, before antigen retrieval in citrate buffer pH 6.0 (\#S1699 Dako) at $121^{\circ} \mathrm{C}$ for 10 minutes. Next, the slides were cooled for 20 minutes before washing in $1 \times$ wash buffer (\#K8007 Dako). All staining was performed in a Dako Autostainer (Agilent Technologies). Slides were incubated in Dual Endogenous Enzyme Block (\#S2003 Dako) for 10 minutes and in protein-free blocking solution (\#X0909 Dako) for 20 minutes, followed by the appropriate dilution of primary antibody and incubated for 60 minutes at room temperature. The following primary antibodies were 


\section{Table 1. Overview of Patient Tumor and Treatment Characteristics}

\begin{tabular}{|c|c|c|c|}
\hline & $\begin{array}{l}\text { AGM } \\
\%(n)\end{array}$ & $\begin{array}{c}\text { Non-AGM } \\
\%(n)\end{array}$ & $P$ Value \\
\hline Patients, $\mathrm{n}$ & 68 & 100 & \\
\hline \multicolumn{4}{|l|}{ Patient characteristics } \\
\hline Male sex & $65 \%(44)$ & $63 \%(63)$ & .82 \\
\hline Age at melanoma diagnosis, mean [SD] & 55 [13] & $55[14]$ & .97 \\
\hline Age at start of $\mathrm{ICl}$, mean [SD] & 59 [12] & $58[14]$ & .90 \\
\hline Follow-up in years from start ICl, median (IQR) & $1.7(0.63-4.5)$ & $4.7(2.9-6.4)$ & $<.001$ \\
\hline Deceased & $71 \%(48)$ & $37 \%(37)$ & $<.001$ \\
\hline \multicolumn{4}{|l|}{ Tumor characteristics } \\
\hline \multicolumn{4}{|l|}{ Primary melanoma subtype } \\
\hline Cutaneous & $50 \%(34)$ & $70 \%(70)$ & NS \\
\hline Unknown primary & $34 \%(23)$ & $17 \%(17)$ & .021 \\
\hline Other & $21 \%(11)$ & $13 \%(13)$ & NS \\
\hline Mucosal & $10 \%(7)$ & $5 \%(5)$ & \\
\hline Ocular & $6 \%(4)$ & $6 \%(6)$ & \\
\hline Acral & $0 \%(0)$ & $2 \%(2)$ & \\
\hline Stage at diagnosis & & & $<.001$ \\
\hline 1 & $7 \%(5)$ & $18 \%(18)$ & \\
\hline II & $32 \%(22)$ & $26 \%(26)$ & \\
\hline III & $26 \%(18)$ & $44 \%(44)$ & \\
\hline IV & $24 \%(23)$ & $12 \%(12)$ & \\
\hline \multicolumn{4}{|l|}{ Mutation status, number/number tested (\%) } \\
\hline BRAF & $30 / 66(45 \%)$ & $33 / 99(39 \%)$ & .12 \\
\hline NRAS & $13 / 39(33 \%)$ & $14 / 86(16 \%)$ & .032 \\
\hline NF1 & $1 / 21(5 \%)$ & $13 / 54(24 \%)$ & .095 \\
\hline$c-K I T$ & $1 / 47(2 \%)$ & $5 / 88(6 \%)$ & .66 \\
\hline M-stage at time of $\mathrm{ICl}$ initiation & & & .36 \\
\hline Mo & $3 \%(3)$ & $6 \%(6)$ & \\
\hline M1a & $16 \%(11)$ & $22 \%(22)$ & \\
\hline M1b & $18 \%(12)$ & $25 \%(25)$ & \\
\hline M1c & $32 \%(22)$ & $23 \%(23)$ & \\
\hline M1d & $31 \%(21)$ & $24 \%(24)$ & \\
\hline Baseline LDH & & & .47 \\
\hline$\leq 1 \times$ ULN $^{a}$ & $65 \%(44)$ & $69 \%(69)$ & \\
\hline$>1 \times$ ULN & $26 \%(18)$ & $19 \%(19)$ & \\
\hline Unknown & $9 \%(6)$ & $12 \%(12)$ & \\
\hline Number of involved organ systems, mean [SD] & $3.7[2.3]$ & $2.7[1.5]$ & $<.001$ \\
\hline Metastatic burden (organ systems involved, categorical) & & & .57 \\
\hline 1 & $21 \%(14)$ & $25 \%(25)$ & \\
\hline 2 & $22 \%(15)$ & $26 \%(26)$ & \\
\hline$\geq 3$ & $57 \%(39)$ & $49 \%(49)$ & \\
\hline \multicolumn{4}{|l|}{ Prevalent metastatic sites } \\
\hline Lung & $78 \%(53)$ & $71 \%(71)$ & NS \\
\hline Liver & $50 \%(34)$ & $30 \%(30)$ & .009 \\
\hline Brain & $46 \%(31)$ & $43 \%(43)$ & NS \\
\hline
\end{tabular}




\begin{tabular}{|c|c|c|c|}
\hline & $\begin{array}{l}\text { AGM } \\
\%(n)\end{array}$ & $\begin{array}{c}\text { Non-AGM } \\
\%(n)\end{array}$ & $P$ Value \\
\hline \multicolumn{4}{|c|}{ Time to nonadrenal metastatic sites in years, median (range) } \\
\hline Lung & $2.36(0-16.53)$ & $1.75(0-29.6)$ & NS \\
\hline Liver & $2.51(0-16.03)$ & $2.76(0-29.6)$ & NS \\
\hline Brain & $1.98(0-16.53)$ & $2.04(0-15.67)$ & NS \\
\hline \multicolumn{4}{|l|}{ Treatment } \\
\hline Immunotherapy ${ }^{\mathrm{b}}$ & $100 \%(68)$ & $100 \%(100)$ & \\
\hline Immunotherapy regimen & & & .075 \\
\hline Anti-CTLA-4 monotherapy & $29 \%(20)$ & $20 \%(20)$ & \\
\hline Anti-PD-L1 monotherapy & $19 \%(13)$ & $17 \%(17)$ & \\
\hline Combination therapy & $22 \%(15)$ & $14 \%(14)$ & \\
\hline Sequential therapy & $29 \%(20)$ & $49 \%(49)$ & \\
\hline Molecular targeted therapy ${ }^{c}$ & $25 \%(17)$ & $33 \%(33)$ & .27 \\
\hline Chemotherapy $^{\mathrm{d}}$ & $28 \%(19)$ & $23 \%(23)$ & .47 \\
\hline
\end{tabular}

Abbreviations: AGM, adrenal gland metastasis; ICl, immune checkpoint inhibitor; IOR, interquartile range; LDH, lactate dehydrogenase; NS, not significant; ULN, upper limit of normal.

aLDH ULN is defined as $\leq 271 \mathrm{U} / \mathrm{L}$, determined by the treating clinical laboratory.

bImmunotherapies include ipilimumab, pembrolizumab, nivolumab, atezolizumab, bevacizumab, IDO1 inhibitors, anti-LAG3, IFN- $\alpha$, and IL-2.

'Molecular targeted therapies include dabrafenib, trametinib, cobimetinib, vemurafenib, regorafenib, insulin-like growth factor 1 inhibitors, apomab, and $\mathrm{Pl}-88$.

${ }^{d}$ Chemotherapy drugs include cisplatin, vinblastine, dacarbazine, carmustine, paclitaxel, capecitabine, temozolomide, and vincristine sulfate liposome.

used: CD8 (Thermo \#MS-457-S1) 1:50 and CD45 (DAKO \#M0701) 1:100. Slides were developed using MACH 2 Mouse AP-Polymer (\#RALP521L Biocare Medical). Slides were then incubated in Vulcan Fast Red (VFR; \#FR805S BioCare Medical) for 15 minutes. Slides were washed using $1 \times$ wash buffer after incubation with each reagent and with $\mathrm{dH}_{2} \mathrm{O}$ after incubation with VFR. Slides were counterstained with hematoxylin (\#S3301 Dako) for 10 minutes. Finally, slides were scanned and imaged using the Aperio eSlide Manager and ImageScope system (Leica).

\section{Statistical Analysis}

Patient, tumor, and treatment characteristics were compared between patients with and without an AGM. Continuous variables were expressed by means and standard deviations, and categorical variables were expressed as absolute numbers and percentages. Differences between the groups were assessed using Fisher exact test and Student $t$ test for categorical and continuous variables, respectively.

ICI response rates were defined as complete response (CR) and partial response (PR) versus stable disease (SD) and progressive disease (PD). Disease control rate (DCR) was defined as $\mathrm{CR}$, PR, and SD versus $\mathrm{PD}$. Overall response rate was defined as $\mathrm{CR}$ and $\mathrm{PR}$ versus SD and PD. For patients with an AGM, the response and disease control rates were calculated for adrenal and nonadrenal gland sites. Response rates and disease control rates were compared between patients with and without an AGM using Fisher exact tests. Disease-specific survival was calculated as the duration from ICI start date until either death resulting from disease progression or last follow-up. Kaplan-Meier curves were used for disease-specific survival plots with $P$ values generated from log-rank tests. A multivariable Cox-proportional hazards model controlling for patient age, sex, site of primary melanoma, and $\mathrm{M}$ stage was used to evaluate the association of the presence of an AGM with disease-specific survival.

A 2-sided significance level of .05 was used for all statistical tests. All analyses were performed using STATA, version 15.1 (StataCorp LLP). Figures were generated in either STATA version 15.1 or GraphPad Prism 6.07 (GraphPad Software).

\section{Results}

\section{Sociodemographic and Clinical Characteristics}

A total of 68 patients met the criteria of metastatic malignant melanoma with metastasis to one or both adrenal glands who received at least one dose of immune checkpoint blockade and had adequate follow-up information. Mean age at initial ICI treatment was 59 years (range, 26-91 years), compared with 58 years (range, 25-88 years) in the control group of 100 patients with stage IV disease without AGM. The male/female ratio was $65 \%$ male, compared with $63 \%$ in the non-AGM group. In the 
AGM group, $50 \%$ had a cutaneous primary melanoma, $10 \%$ mucosal, and $6 \%$ ocular, and $34 \%$ had an unknown primary. In the non-AGM group these were $70 \%, 5 \%, 6 \%$, and $17 \%$, respectively. Although not a part of our inclusion or exclusion criteria, all patients in this study had an ECOG performance status of 0 or 1 at the time of treatment initiation. Patients diagnosed with an AGM were more likely to be initially diagnosed with stage IV disease compared with the non-AMG group (Table 1).

Of the patients with an AGM, the median time from melanoma diagnosis to AGM was 2.2 years (range, 0-16 years), and the median time from AGM diagnosis to death was 0.80 years (range, $0.011-8.0$ years). Of the 68 patients, 20 had a metastasis in the left adrenal gland, 22 in the right adrenal gland, 25 bilateral, and 1 unknown. A total of 9 patients underwent an adrenalectomy, of which 1 was bilateral. Median time from AGM diagnosis to surgery was 6.5 months (range, 0.69-33 months) (Table 2).

In the non-AGM group, $71 \%(n=71)$ of patients were diagnosed with lung metastases, $30 \%(n=30)$ with liver metastases, and $43 \%(n=43)$ with brain metastases compared with $78 \%(n=53), 50 \%(n=34)$, and $46 \%(n=31)$, respectively, in the AGM group (Table 1). No statistically significant differences were seen in the median time to liver, lung, or brain metastases between the 2 groups (Table 1). Furthermore, there was no statically significant difference between the median time to AGM compared with liver, lung, or brain metastases (Tables 1 and 2).
We observed an increased frequency of NRAS mutations in the AGM group $(n=13 ; 33 \%)$ compared with the non-AGM group $(\mathrm{n}=14 ; 16 \%)(P=.032)$, and a trend toward a decreased frequency of $N F 1$ mutations in patients with AGM $(\mathrm{n}=1 ; 5 \%)$ compared with those without $(\mathrm{n}=13 ; 24 \%)(P=.095)$ (Table 1$). B R A F$ mutations were present in $45 \%$ and $c$-KIT mutations in $2 \%$ of the patients with AGM compared with $39 \%$ and $6 \%$, respectively, in those without. Levels of circulating lactate dehydrogenase (LDH) and the frequency of patients with elevated $\mathrm{LDH}$ levels at time of diagnosis were similar between the groups (Table 1). Patients diagnosed with an AGM had an increased average number of involved organ systems (3.7) at ICI initiation compared with the non-AGM group (2.7; $P<.001$ ) (Table 1).

All patients included in this study received at least 1 dose of an ICI. Although there were no differences in the frequency of patients receiving either anti-CTLA-4 or anti-PD-1 monotherapy, more patients with AGM $(22 \%)$ received combination treatment than those without (14\%), whereas more patients without AGM (49\%) received both therapies at separate (sequential) timepoints than those with AGM (29\%). Notably, many of these patients also received other forms of immunotherapy, in total up to 4 lines. Moreover, 17 (25\%) patients in the group with AGM and 33 (33\%) in the group without AGM received a form of molecular targeted therapy, and 19 (28\%) and 23 (23\%) received chemotherapy, respectively (Table 1).

\begin{tabular}{|c|c|c|}
\hline Characteristic & AGM & Non-AGM \\
\hline Time from diagnosis to AGM, median (range), y & $2.2(0-16)$ & \\
\hline Time from AGM to death, median (range), y & $0.80(0.011-8.0)$ & \\
\hline \multicolumn{3}{|l|}{ Side of AGM (n) } \\
\hline Left & $29 \%(20)$ & \\
\hline Right & $32 \%(22)$ & \\
\hline Bilateral & $37 \%(25)$ & \\
\hline Unknown & $1 \%(1)$ & \\
\hline \multicolumn{3}{|l|}{ Surgery } \\
\hline Patients who underwent surgery, $\mathrm{n}$ & 9 & \\
\hline Resection of left adrenal gland, $\mathrm{n}$ & 9 & \\
\hline Resection of right adrenal gland, $\mathrm{n}$ & 1 & \\
\hline Time from AGM to surgery, median (range), mo & $6.5(0.69-33)$ & \\
\hline \multicolumn{3}{|l|}{ Treatment response } \\
\hline \multicolumn{3}{|l|}{ Best responses ( $n$ ) } \\
\hline Complete response & $10 \%(7)$ & $10 \%(10)$ \\
\hline Partial response & $6 \%(4)$ & $45 \%(45)$ \\
\hline Stable disease & $13 \%(9)$ & $21 \%(21)$ \\
\hline Progressive disease & $71 \%(48)$ & $24 \%(24)$ \\
\hline
\end{tabular}

Abbreviation: AGM, adrenal gland metastasis. 


\section{Response to $\mathrm{ICls}$}

We compared the response rates of the AGM versus other metastatic sites in patients with an AGM, as well as the responses in patients without AGM. We observed that the DCR (CR, PR, and SD) for the AGM was $29 \%$, whereas in these same patients the non-AGM had a DCR of $46 \%$ $(P<.001)$ (Figure $1 \mathrm{~A}$ and $1 \mathrm{~B})$. This was significantly lower than in the patients without an AGM, in whom the DCR was $76 \%(P<.001)$ (Figure 1C). Similarly, the overall response rate $(\mathrm{CR}, \mathrm{PR})$ was $16 \%$ in AGMs, $22 \%$ in the nonAGMs from these same patients, and $55 \%$ in the patients without AGM $(P=.011)$ (Figure 1$)$.

\section{Survival}

Median follow-up from the start of ICI therapy was 1.7 years in the AGM group and 4.7 years in the non-AGM group. Of the patients with an AGM, $71 \%$ were deceased, compared with $37 \%$ in the non-AGM group $(P<.001$; Table 1). Using a multivariable Cox proportional-hazards model controlling for age, sex, primary melanoma type, pretreatment $\mathrm{LDH}$ level, presence of liver metastases, number of involved organ systems, and immunotherapy regimen, we found that patients diagnosed with an AGM had a significantly decreased OS (median OS, 3.1 years from start of initial ICI therapy) compared with those without an AGM (median OS, not reached; hazard ratio [HR], 2.96; 95\% CI, 1.8-4.8; $P<.001$ ) (Figure 2A). A similar observation was made comparing survival of the 2 groups from the time of anti-PD-1 initiation, considering that anti-PD-1 therapy has a survival benefit over singleagent anti-CTLA- $4{ }^{22}$ This showed that patients diagnosed with an AGM had a significantly decreased OS (median OS, 2.7 years from start of anti-PD-1 treatment) compared with those without an AGM (median OS, not reached; HR, 3.12; 95\% CI, 1.8-5.4; $P<.001$ ) (Figure 2B). Additionally, we compared survival between the $2 \mathrm{popu}-$ lations from the time of initial melanoma diagnosis and found that patients diagnosed with an AGM had a median OS of 5.6 years, compared with 13.9 years in patients without an AGM (HR, 2.38; 95\% CI, 1.5-3.8; $P<.001$ ) (Figure 2C). Finally, we compared survival between the 2 populations from the time of stage IV diagnosis and found that patients diagnosed with an AGM had a median OS of 3.6 years, compared with 11.9 years in patients without an AGM (HR, 2.96; 95\% CI, 1.8-4.8; $P<.001$ ) (Figure 2D). Further illustrating the negative impact of metastases to the adrenal gland, we found that the presence of an AGM was associated with worse OS compared with lung, liver or brain metastases when analyzed on their own (supplemental eFigure 1, available with this article at JNCCN.org).

\section{Surgical Resection of AGM}

Nine patients who had an AGM remaining as the only site of PD underwent an adrenalectomy (Table 2). Although not statistically significant $(P=.052)$, univariate analysis showed that the median OS was not reached for the 9 patients who underwent surgical resection of the AGM, where it was 1.5 years for those in whom the AGM was not resected (Figure 3A).

\section{Tumor Microenvironment}

To gain an understanding of the possible mechanisms of resistance in AGM, we performed immunohistochemical
A

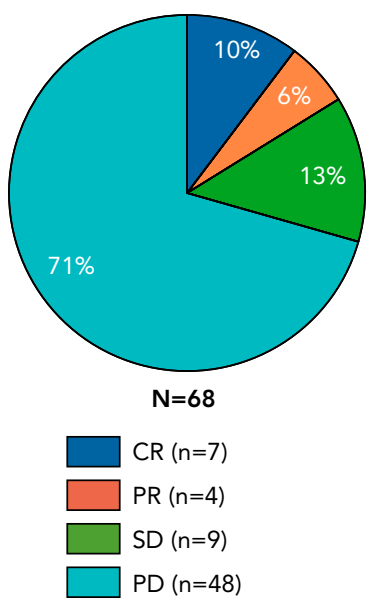

B

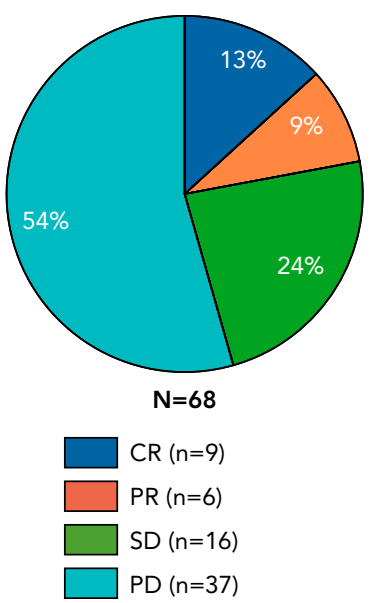

C

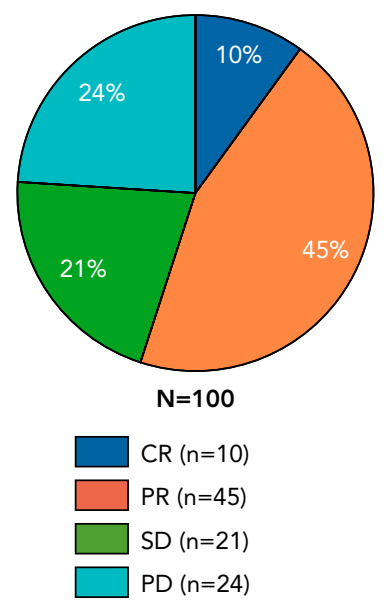

Figure 1. Comparison of responses to immune checkpoint inhibitor therapy. Distribution of response rates are shown for ( $A) A G M$ and (B) other metastatic sites of patients with $A G M$, and for $(C)$ metastatic sites of patients without AGM. All responses were determined by a consensus between the radiologist and the treating medical oncologist according to RECIST 1.1 criteria.

Abbreviations: AGM, adrenal gland metastasis; $C R$, complete response; SD, stable disease; PD, progressive disease; PR, partial response. 
A

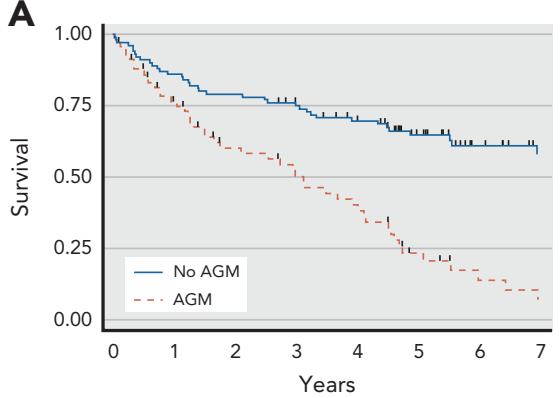

Number at risk

$\begin{array}{rrrrrrrrr}\text { No AGM } & 100 & 86 & 79 & 71 & 61 & 43 & 27 & 20 \\ \text { AGM } & 68 & 44 & 31 & 25 & 20 & 9 & 4 & 2\end{array}$

\section{B}
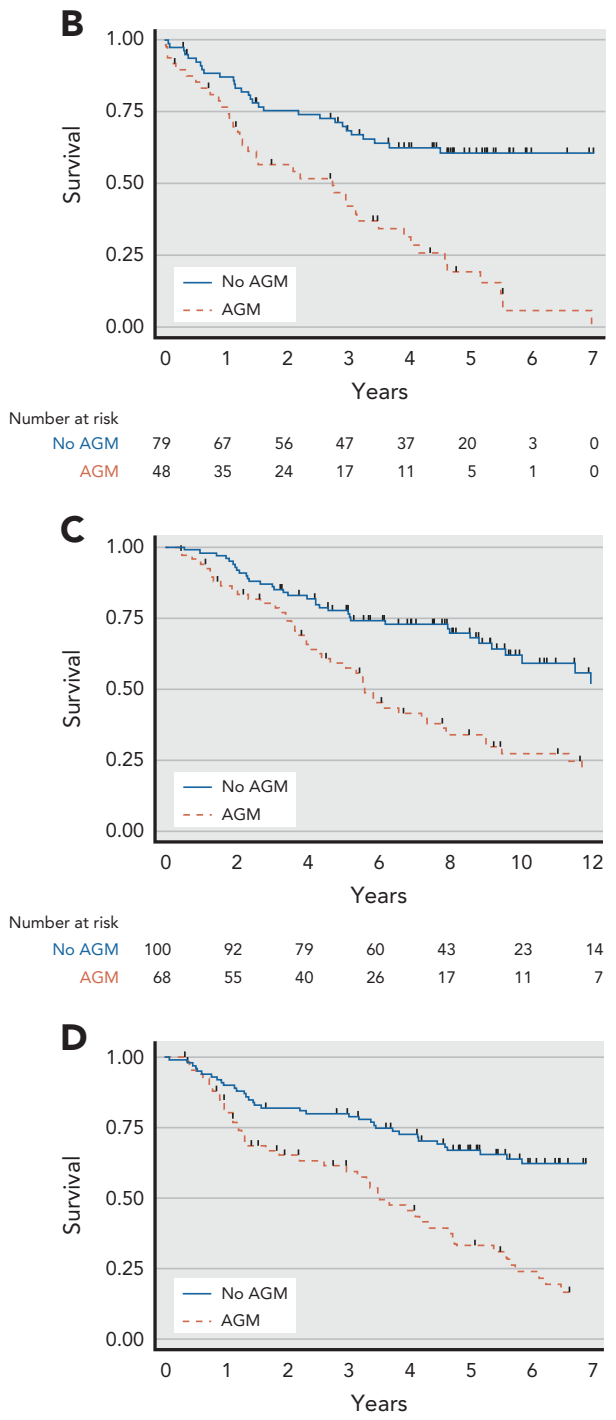

Number at risk

$\begin{array}{rrrrrrrrr}\text { No AGM } & 100 & 90 & 81 & 76 & 67 & 49 & 36 & 26 \\ \text { AGM } & 68 & 51 & 36 & 30 & 23 & 16 & 10 & 6\end{array}$
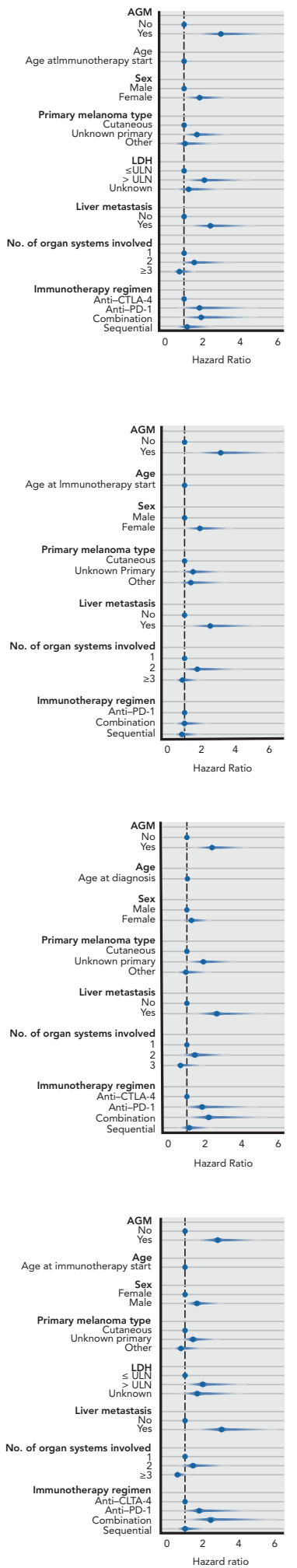

Figure 2. Overall survival and hazard ratio analysis. Kaplan-Meier curves and associated forest plots showing survival for patients with $A G M$ and without AGM, and matching forest plots with the hazard ratios per patient characteristic from the (A) start of start ICl, (B) start of anti-PD-1 treatment, (C) time of initial melanoma diagnosis, and (D) time of stage IV diagnosis. Errors bars on the forest plot indicate $95 \%$ confidence intervals.

Patients were censored at the time of last follow-up.

Abbreviations: AGM, adrenal gland metastasis; ICl, immune checkpoint inhibitor; ULN, upper limit of normal. 
staining on 9 surgically resected ICI-resistant melanoma AGMs. Based on the observed low response rates in AGMs, it was expected that these tumors would exhibit low numbers of tumor-infiltrating immune cells. However, we observed robust infiltration of total immune cells (CD45+ cells) and CD8 $+\mathrm{T}$ cells in all resected specimens (Figure 3B, supplemental eFigures 2-4). Furthermore, we observed that the metastatic melanoma cells were present in the medulla of all 9 samples with evidence of progression into the cortex, where we observed that the tumor eventually replaced most of the normal adrenal gland tissue.

\section{Discussion}

The introduction of ICIs has made a significant impact on survival rates for many patients with a broad range of tumors. However, as previous case reports and studies have shown, AGMs may present an obstacle for successful treatment of patients with advanced-stage disease. ${ }^{11,18,23,24} \mathrm{We}$ and others have hypothesized that the adrenal gland may serve as a so-called sanctuary site, as defined by their inherent immunosuppressive microenvironment, allowing the tumor to grow in an immunologically permissive milieu and limiting the efficacy of ICIs. To further investigate this, we performed a large, single-center, retrospective analysis of all patients with stage IV melanoma with and without an AGM who were treated with ICIs. A total of 68 patients with an AGM and a well-matched control group of 100 patients with stage IV disease without an AGM were included. Our data showed that patients with an AGM had a lower median OS and inferior clinical responses in other metastatic sites compared with patients with stage IV melanoma without an AGM. This is despite a robust immune cell infiltrate in the AGMs. Therefore, this study presents additional evidence suggesting that the adrenal gland provides a potential sanctuary site for melanoma metastases and acts as a potential indicator of patients with more aggressive tumors.

The efficacy of antitumor immune responses induced by ICIs relies on the initiation and persistence of a cascade of antitumor effector mechanisms. ${ }^{25,26}$ Factors within the tumor microenvironment influence the effectiveness of these mechanisms. ${ }^{25,26}$ Several mediators, including cytokines and growth factors released by tumor cells, as well as by various immune cells that promote tumor progression (ie, regulatory $\mathrm{T}$ cells and myeloid-derived suppressor cells), create an immunosuppressive state within the tumor, leading to a hostile tumor microenvironment. ${ }^{27-30}$ It is likely that this immunosuppressive milieu plays an even more prominent role in the immunoresistance of AGMs,

\section{A}

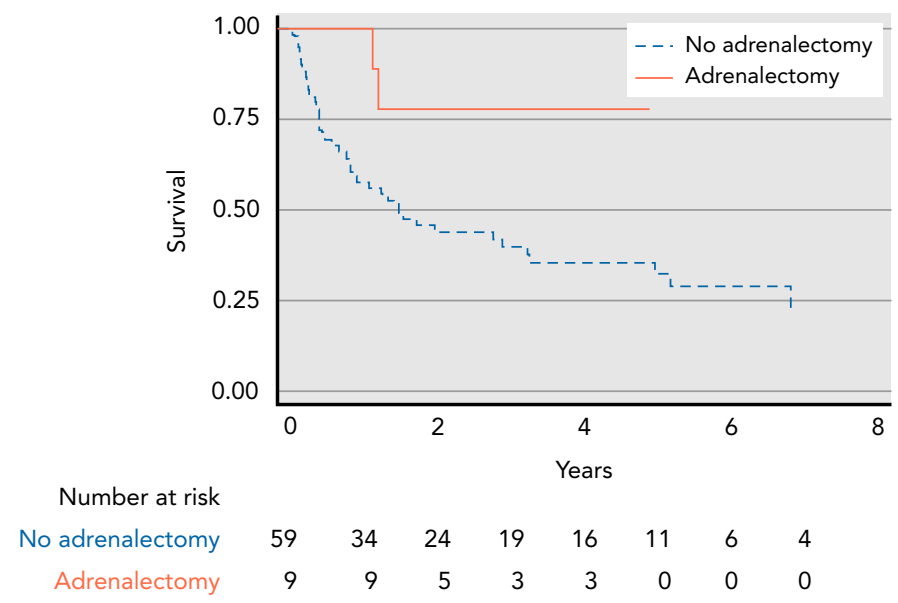

B

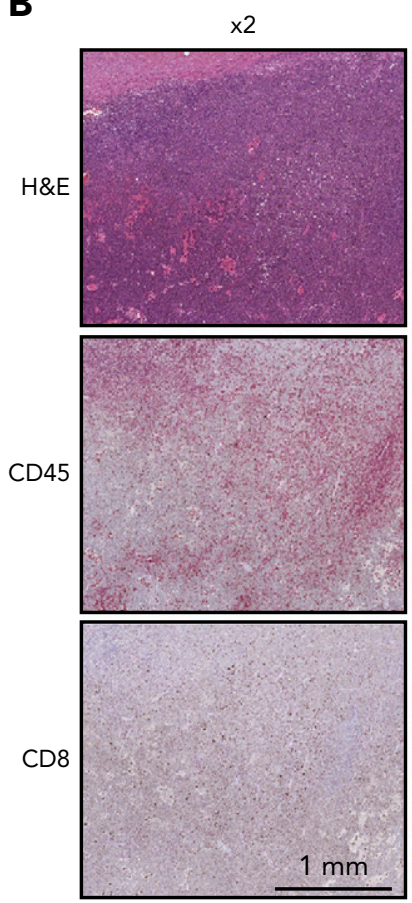

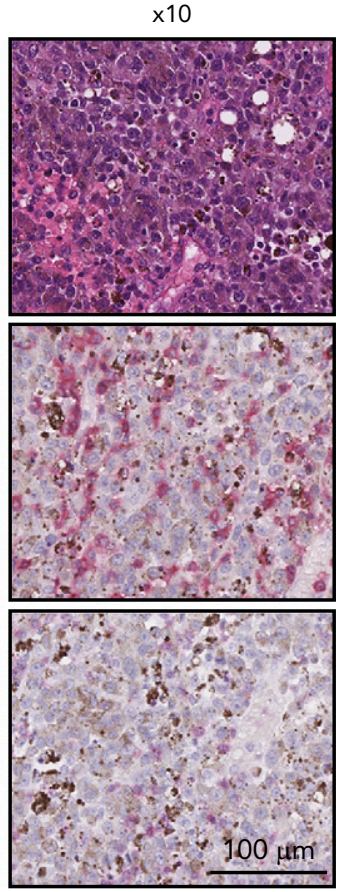

Figure 3. Overall survival after adrenalectomy and analysis of the adrenal metastasis microenvironment. (A) Kaplan-Meier curve showing overall survival of patients with melanoma from the diagnosis of AGM. (B) Representative images showing H\&E, CD45 (total immune cells), and CD8 (CD8 + T cells) staining rom serial sections in the same location of one resected AGM (original magnification: $\times 2$ and $\times 10$ ). For CD45 and CD8, red staining indicates positivity.

Abbreviations: AGM, adrenal gland metastasis; H\&E, hematoxylin-eosin. 
limiting the efficacy of ICIs within the adrenal gland. Considering the important function of the adrenal gland in corticosteroid production, ${ }^{19,20}$ it is possible that this creates an ideal immunosuppressive environment for the metastatic cells to escape immune surveillance and continue proliferating. Provided that cancer immunotherapies do not directly target tumor cells, but rather target receptors and ligands involved in the regulation of immune cells, their efficacy relies solely on the patient's own immune system to clear the tumor cells. This is one of several possible explanations for why melanoma metastases to the adrenal gland are highly resistant to ICIs.

Other studies have proposed different histologic tumor microenvironment phenotypes based on the amount of immune cell infiltration. To investigate whether histologic findings would support this hypothesis, we examined 9 melanoma AGMs from 8 patients for expression of CD45 and CD8. We expected to find low levels of immune cell infiltration. Contrary to our expectations, we observed brisk CD45+ immune cell infiltration in the AGMs. We further observed significant CD8+ T-cell infiltration in several samples. The finding of a brisk immune cell infiltrate supports the possibility that there are other intrinsic factors contributing to the immunotherapy resistance within the metastatic tumor microenvironment in the adrenal gland. Unraveling these factors may lead to the possibility of designing additional therapies to improve ICI responses in the adrenal gland.

Previous studies have observed that the presence of certain metastatic sites or baseline characteristics correlates with differential outcomes in patients with melanoma, including metastases to liver, ${ }^{31,32}$ lung, ${ }^{8,9}$ or brain ${ }^{33,34}$; circulating levels of $\mathrm{LDH}^{35,36}$; and number of metastatic sites. ${ }^{37,38}$ To determine whether the presence of an AGM may help further classify these prognostic indicators, we compared OS based on these well-established indicators and on the presence of AGM. Controlling for the negative impact of these clinical factors, we found that the presence of an AGM was independently associated with worse OS. Likewise, the time between initial melanoma diagnosis and the diagnosis of adrenal, lung, liver, or brain metastases was not statistically significantly different within the AGM group or compared with the patients without AGM, indicating that AGM development itself is not correlated with end-stage metastatic disease. Together, these observations suggest that AGMs may be indicative of tumors with a high metastatic potential. However, further investigation is required to establish this.

Differences in site-specific ICI responses have been observed in other cancers, such as in lung cancer, where metastases to the adrenal gland have also been shown to be more resistant to anti-PD-1 therapy. ${ }^{11}$ Likewise, when considering primary tumors of the adrenal gland, treatment with immunotherapies has similar disappointing results. ${ }^{39}$ Cosentini et $\mathrm{al}^{39}$ proposed an interesting model to explain how tumor intrinsic and systemic factors could impair immunotherapy efficacy in adrenocortical carcinoma (ACC). According to their model, the combination of low PD-L1 expression (a predictor of activity and survival in patients treated with ICIs for some tumors), local immunosuppressive hormone secretion, and mutations in several pathways that impair CD8+ T-cell function leads to immunoresistance in ACC. ${ }^{39,40}$ Further research is needed to determine whether their model can be extrapolated to patients with melanoma and an AGM.

Comparisons of intrinsic and pathologic variables among melanoma tumors have revealed several prognostic factors that are associated with differential outcomes. $^{25,26,41,42}$ We observed several important differences between the 2 cohorts in this study. First, we noted an increased abundance of melanomas of unknown primary in the AGM group. Patients diagnosed with a melanoma of unknown primary are generally diagnosed at a more advanced disease stage and have worse outcomes compared with the general melanoma population. ${ }^{43}$ Second, clinical molecular analysis revealed an increased frequency of NRAS mutations and a trend toward decreased NF1 mutations among the AGM patient cohort. ${ }^{41}$ This is the first study reporting an increased abundance of NRAS mutations in patients with melanoma diagnosed with an AGM, and supports the further development of therapies targeting RAS-driven tumors. However, there are conflicting reports about the role these mutations play in responses to immunotherapy, limiting our ability to adequately interpret these results. ${ }^{41,42}$

The tissue-specific microenvironment of the adrenal gland poses several challenges to successful antitumor immunity. The locally produced glucocorticoids have well-described immunosuppressive activity. ${ }^{44}$ In addition to suppressing lymphocyte proliferation and function, glucocorticoids specifically upregulate $\beta$-catenin, promoting immune cell exclusion from the tumor microenvironment. ${ }^{45}$ Furthermore, glucocorticoids slow dendritic cell maturation and decrease the expression of major histocompatibility complex class I and II, limiting the ability of $\mathrm{T}$ cells to recognize tumor cells. ${ }^{46-48}$ Other factors produced in the adrenal gland, such as catecholamines and androgens, exert further potent immunosuppressive functions, including blocking immune cell proliferation, cytokine production, and cytotoxic capacity. ${ }^{49,50}$ Together, these factors make the adrenal gland a particularly hostile site for the establishment of effective antitumor immunity. However, it is important to note that this was not directly tested in this study and that it remains a possibility that as the tumor progresses and replaces normal adrenal tissue with tumor cells, normal adrenal function will be lost.

Likewise, corticosteroids have been shown to have similar direct effects on melanoma cells. In vitro studies 
with melanoma cell lines have found that glucocorticoids prevent chemotherapy-induced apoptosis. ${ }^{51}$ Paradoxically, mouse models of melanoma show that glucocorticoids decrease melanoma cell proliferation in vivo. ${ }^{52}$ This suggests that the tumor microenvironment of the adrenal gland may promote a less proliferative and more invasive phenotype of tumor cells, further supporting the possibility that the presence of an AGM may be indictive of tumors with a high metastatic potential. ${ }^{52,53}$

Before the use of CT as a routine imaging technique, AGMs were rarely diagnosed before death. Improved imaging techniques have increased the ability to diagnose AGM earlier in melanoma progression, potentially broadening the treatment window for targeting these metastases. In 1991 , Branum et $\mathrm{al}^{18}$ was the first to report a potential survival benefit for patients with metastatic melanoma to one or both adrenal glands who underwent a surgical intervention compared with nonsurgical treatment. This finding is further supported by a report from Zippel et $\mathrm{al}^{23}$ showing that minimally invasive adrenalectomy is safe and improves survival compared with conservative management in a selected group of patients with metastatic melanoma with operable AGMs. Moreover, a recent review from Spartalis et $\mathrm{al}^{24}$ that included studies from patients with different primary tumors, including melanoma, concludes that an adrenalectomy should be performed when the lesion is isolated in the adrenal gland and the site of primary cancer has or can be resected, because this procedure has the potential to offer prolonged survival. Although our study was underpowered to perform this analysis due to a low number of patients undergoing an adrenalectomy, the strong trend toward improved survival for patients who underwent surgical resection of AGMs corroborates these previous findings. However, it is important to note that this difference in survival may reflect selection bias that is confounded by several factors relating the amenability for surgery, response to therapy, and disease burden, which should be discussed by multidisciplinary teams on a caseby-case basis. Our current practice is to proceed with adrenalectomy in patients treated with ICI in whom the adrenal gland is the only remaining site of PD. Further studies in patients with melanoma and an AGM treated with ICIs will determine whether this is the optimal approach.

This study should be interpreted in light of its limitations. First, this is a single-center study, meaning that there may be inherent bias in a tertiary recruitment population. Second, due to the heterogeneity of the patients and the treatments they received, this is an observational study and no definitive treatment recommendations can be made at this time. Furthermore, some patients in the control group may have had occult AGMs, considering that approximately $50 \%$ of autopsied patients were found to have an AGM, and most of these patients did not undergo an autopsy or have had a CT scan shortly before they died. However, despite these limitations, this is, to our knowledge, the first study that examines the survival of a large group of patients with melanoma and an AGM who were treated with ICIs, and these data significantly expand our knowledge about the prognosis of patients with an AGM. Although the mechanisms accounting for the treatment resistance of AGMs remain to be fully elucidated, the results of this study are likely to be important and informative for treating physicians when deciding the best treatment plan for individual patients.

\section{Conclusions}

In our cohort of patients with melanoma, those diagnosed with an AGM had lower ICI response rates and OS. These results suggest that tissue-specific microenvironments of AGMs present unique challenges that may require novel adrenal gland-directed therapies or surgical resection.

Submitted June 1, 2020; final revision received December 15, 2020; accepted for publication December 16, 2020

Published online August 4, 2021.

Author contributions: Study concept and design: All authors. Data acquisition: All authors. Data analysis and interpretation: All authors. Manuscript preparation: All authors. Final approval: All authors.

Disclosures: Dr. Lewis has disclosed receiving grant/research support and personal fees from Roche/Genentech. Dr. McCarter has disclosed receiving grant/research support from Merck \& Co., Inc. Dr. Mushtaq has disclosed owning stock and having other ownership interests in Abbott Laboratories, Amgen, Boston Scientific, Bristol Myers Squibb, Celgene Corp Company, Edwards Lifesciences, Gilead Sciences, Johnson \& Johnson, and Medtronic The remaining authors have disclosed that they have not received any financial considerations from any person or organization to support the preparation, analysis, results, or discussion of this article.

Funding: This work was supported in part by funding from the Patten-Davis Foundation, the Moore Family Foundation, the University of Colorado Department of Surgery Academic Enrichment Fund, University of Colorado Cancer Center Support Grant (P30CA046934), and the Cancer League of Colorado.

Correspondence: William A. Robinson, MD, PhD, Center for Rare Melanomas, University of Colorado Anschutz Medical Campus, 12801 East 17th Avenue, MS8117, Aurora, CO 80045.

Email: william.robinson@cuanschutz.edu

\section{References}

1. National Cancer Institute Surveillance, Epidemiology, and End Results Program. Cancer Stat Facts: Melanoma of the Skin. Accessed June 1 2019. Available at: https://seer.cancer.gov/statfacts/html/melan.html

2. Topalian SL, Hodi FS, Brahmer JR, et al. Safety, activity, and immune correlates of anti-PD-1 antibody in cancer. N Engl J Med 2012;366: 2443-2454.

3. Brahmer JR, Tykodi SS, Chow LQ, et al. Safety and activity of anti-PD-L1 antibody in patients with advanced cancer. N Engl J Med 2012;366:2455-2465.

4. Rijnders M, de Wit R, Boormans JL, et al. Systematic review of immune checkpoint inhibition in urological cancers. Eur Urol 2017;72:411-423.

5. Larkin J, Chiarion-Sileni V, Gonzalez R, et al. Combined nivolumab and ipilimumab or monotherapy in untreated melanoma. N Engl J Med 2015;373:23-34.

6. Ribas A, Hamid O, Daud A, et al. Association of pembrolizumab with tumor response and survival among patients with advanced melanoma. JAMA 2016;315:1600-1609. 
7. Robert C, Schachter J, Long GV, et al. Pembrolizumab versus ipilimumab in advanced melanoma. N Engl J Med 2015;372:2521-2532.

8. Lee JHJ, Lyle M, Menzies AM, et al. Metastasis-specific patterns of response and progression with anti-PD-1 treatment in metastatic melanoma. Pigment Cell Melanoma Res 2018;31:404-410.

9. Khoja L, Kibiro M, Metser U, et al. Patterns of response to anti-PD-1 treatment: an exploratory comparison of four radiological response criteria and associations with overall survival in metastatic melanoma patients. Br J Cancer 2016;115:1186-1192.

10. Tumeh PC, Harview CL, Yearley JH, et al. PD-1 blockade induces responses by inhibiting adaptive immune resistance. Nature 2014; 515:568-571

11. Nguyen MC, Shah MH, Liebner DA, et al. The adrenal gland as a sanctuary site of metastases after pembrolizumab treatment: a case series. $J$ Natl Compr Canc Netw 2018;16:1279-1283

12. Patel JK, Didolkar MS, Pickren JW, et al. Metastatic pattern of malignant melanoma. A study of 216 autopsy cases. Am J Surg 1978;135:807-810.

13. Cingam SR, Mukkamalla SKR, Karanchi H. Adrenal metastasis. In: StatPearls [Internet]. Treasure Island, FL: StatPearls Publishing LLC; 2020.

14. Kanczkowski W, Sue M, Bornstein SR. The adrenal gland microenvironment in health, disease and during regeneration. Hormones (Athens) 2017;16:251-265.

15. Aftab Z, Wladis A. Skandalakis' surgical anatomy: the embryology and anatomic basis of modern surgery. Sultan Qaboos Univ Med J 2008;8:97-98

16. Ejaz S, Shawa H, Henderson SA, Habra MA. Melanoma of unknown primary origin presenting as a rapidly enlarging adrenal mass [published online June 19, 2013]. BMJ Case Rep, doi: 10.1136/bcr-2013-009727

17. Lam KY, Lo CY. Metastatic tumours of the adrenal glands: a 30-year experience in a teaching hospital. Clin Endocrinol (Oxf) 2002;56:95-101.

18. Branum GD, Epstein RE, Leight GS, et al. The role of resection in the management of melanoma metastatic to the adrenal gland. Surgery 1991;109:127-131.

19. Cain DW, Cidlowski JA. Immune regulation by glucocorticoids. Nat Rev Immunol 2017;17:233-247.

20. Bowen DL, Fauci AS. Adrenal corticosteroids. In: Gallin JI, Goldstein I, Snyderman R, eds. Inflammation: Basic Principles and Clinical Correlates. New York, NY: Raven Press; 1988:877-895.

21. Segal BH, Sneller MC. Infectious complications of immunosuppressive therapy in patients with rheumatic diseases. Rheum Dis Clin North Am 1997:23:219-237.

22. Wolchok JD, Chiarion-Sileni V, Gonzalez R, et al. Overall survival with combined nivolumab and ipilimumab in advanced melanoma. N Engl J Med 2017;377:1345-1356.

23. Zippel $D$, Yalon $T$, Nevo $Y$, et al. The non-responding adrenal metastasis in melanoma: the case for minimally invasive adrenalectomy in the age of modern therapies. Am J Surg 2020;220:349-353.

24. Spartalis E, Drikos I, loannidis A, et al. Metastatic carcinomas of the adrenal glands: from diagnosis to treatment. Anticancer Res 2019;39: 2699-2710.

25. Cogdill AP, Andrews MC, Wargo JA. Hallmarks of response to immune checkpoint blockade. Br J Cancer 2017;117:1-7.

26. Davin P, Toor SM, Sasidharan Nair V, et al. Immune checkpoint inhibitors: recent progress and potential biomarkers. Exp Mol Med 2018;50: $1-11$.

27. Durgeau A, Virk Y, Corgnac S, et al. Recent advances in targeting CD8 Tcell immunity for more effective cancer immunotherapy. Front Immunol 2018;9:14

28. Baumgartner J, Wilson C, Palmer B, et al. Melanoma induces immunosuppression by up-regulating FOXP3(+) regulatory T cells. J Surg Res 2007;141:72-77

29. Tobin RP, Davis D, Jordan KR, et al. The clinical evidence for targeting human myeloid-derived suppressor cells in cancer patients. J Leukoc Biol 2017;102:381-391.

30. Jordan KR, Amaria RN, Ramirez O, et al. Myeloid-derived suppressor cells are associated with disease progression and decreased overall survival in advanced-stage melanoma patients. Cancer Immunol Immunother 2013;62:1711-1722.
31. Tumeh PC, Hellmann MD, Hamid $\mathrm{O}$, et al. Liver metastasis and treatment outcome with anti-PD-1 monoclonal antibody in patients with melanoma and NSCLC. Cancer Immunol Res 2017;5:417-424.

32. Bilen MA, Shabto JM, Martini DJ, et al. Sites of metastasis and association with clinical outcome in advanced stage cancer patients treated with immunotherapy. BMC Cancer 2019;19:857.

33. Gorantla V, Kirkwood JM, Tawbi HA. Melanoma brain metastases: an unmet challenge in the era of active therapy. Curr Oncol Rep 2013;15: 483-491.

34. Spagnolo F, Picasso V, Lambertini M, et al. Survival of patients with metastatic melanoma and brain metastases in the era of MAP-kinase inhibitors and immunologic checkpoint blockade antibodies: a systematic review. Cancer Treat Rev 2016;45:38-45.

35. Diem S, Kasenda B, Spain L, et al. Serum lactate dehydrogenase as an early marker for outcome in patients treated with anti-PD-1 therapy in metastatic melanoma. Br J Cancer 2016;114:256-261.

36. Hopkins AM, Rowland A, Kichenadasse G, et al. Predicting response and toxicity to immune checkpoint inhibitors using routinely available blood and clinical markers. Br J Cancer 2017;117:913-920.

37. Tas F. Metastatic behavior in melanoma: timing, pattern, survival, and influencing factors. J Oncol 2012;2012:647-684.

38. Neuman $\mathrm{HB}$, Patel $\mathrm{A}$, Ishill $\mathrm{N}$, et al. A single-institution validation of the AJCC staging system for stage IV melanoma. Ann Surg Oncol 2008;15:2034-2041.

39. Cosentini D, Grisanti S, Dalla Volta A, et al. Immunotherapy failure in adrenocortical cancer: where next? Endocr Connect 2018;7:E5-8.

40. Hino R, Kabashima K, Kato $Y$, et al. Tumor cell expression of programmed cell death-1 ligand 1 is a prognostic factor for malignant melanoma. Cancer 2010;116:1757-1766.

41. Cirenajwis $H$, Lauss $M$, Ekedahl $H$, et al. NF1-mutated melanoma tumors harbor distinct clinical and biological characteristics. Mol Oncol 2017:11:438-451.

42. Morrison C, Pabla S, Conroy JM, et al. Predicting response to checkpoint inhibitors in melanoma beyond PD-L1 and mutational burden. J Immunother Cancer 2018;6:32.

43. Utter K, Goldman C, Weiss SA, et al. Treatment outcomes for metastatic melanoma of unknown primary in the new era: a single-institution study and review of the literature. Oncology 2017;93:249-258.

44. Ashwell JD, Lu FW, Vacchio MS. Glucocorticoids in T cell development and function*. Annu Rev Immunol 2000;18:309-345.

45. Guan $Y$, Rubenstein NM, Failor KL, et al. Glucocorticoids control betacatenin protein expression and localization through distinct pathways that can be uncoupled by disruption of signaling events required for tight junction formation in rat mammary epithelial tumor cells. Mol Endocrinol 2004;18:214-227

46. Piemonti L, Monti $\mathrm{P}$, Allavena $\mathrm{P}$, et al. Glucocorticoids affect human dendritic cell differentiation and maturation. J Immunol 1999;162:6473-6481.

47. Hunzeker JT, Elftman MD, et al. A marked reduction in priming of cytotoxic CD8+ T cells mediated by stress-induced glucocorticoids involves multiple deficiencies in cross-presentation by dendritic cells. J Immunol 2011;186:183-194.

48. Celada A, McKercher S, Maki RA. Repression of major histocompatibility complex IA expression by glucocorticoids: the glucocorticoid receptor in hibits the DNA binding of the X box DNA binding protein. J Exp Med 1993; 177:691-698

49. Flierl MA, Rittirsch D, Huber-Lang M, et al. Catecholamines-crafty weapons in the inflammatory arsenal of immune/inflammatory cells or opening pandora's box? Mol Med 2008;14:195-204.

50. Gubbels Bupp MR, Jorgensen TN. Androgen-induced immunosuppression Front Immunol 2018;9:794

51. Mattern J, Büchler MW, Herr I. Cell cycle arrest by glucocorticoids may protect normal tissue and solid tumors from cancer therapy. Cancer Biol Ther 2007;6:1345-1354.

52. Bhakoo HS, Paolini NS, Milholland RJ, et al. Glucocorticoid receptors and the effect of glucocorticoids on the growth of B16 melanoma. Cancer Res 1981;41:1695-1701.

53. Gao CF, Xie Q, Su YL, et al. Proliferation and invasion: plasticity in tumor cells. Proc Natl Acad Sci USA 2005;102:10528-10533. 
Supplemental online content for:

\section{Melanoma Metastases to the Adrenal Gland Are Highly Resistant to Immune Checkpoint Inhibitors}

Jessica S.W. Borgers, MD; Richard P. Tobin, PhD; Robert J. Torphy, MD; Victoria M. Vorwald, BS; Robert J. Van Gulick, BS; Carol M. Amato, MS; Dasha T. Cogswell, MS; Tugs-Saikhan Chimed, MS; Kasey L. Couts, PhD; Adrie Van Bokhoven, PhD; Christopher D. Raeburn, MD; Karl D. Lewis, MD; Joshua Wisell, MD; Martin D. McCarter, MD; Rao R. Mushtaq, MD; and William A. Robinson, MD, PhD

J Natl Compr Canc Netw, doi: 10.6004/jnccn.2020.7800

eFigure 1: Survival Analysis Based on Prevalent Nonadrenal Metastatic Sites

eFigure 2: Immunohistochemical Analysis of the Adrenal Metastasis Microenvironment via H\&E Staining eFigure 3: Immunohistochemical Analysis of the Adrenal Metastasis Microenvironment via CD8 Staining eFigure 4: Immunohistochemical Analysis of the Adrenal Metastasis Microenvironment via CD45 Staining 
A

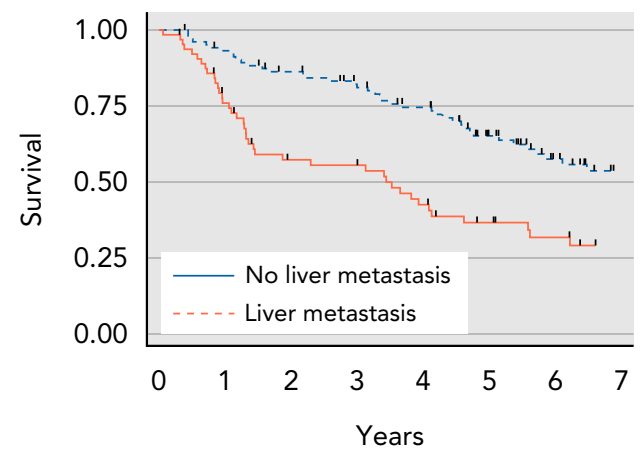

Number at risk

$\begin{array}{rrrrrrrrr}\text { No liver metastasis } & 104 & 95 & 85 & 76 & 67 & 48 & 33 & 23 \\ \text { Liver metastasis } & 64 & 46 & 32 & 30 & 23 & 17 & 13 & 9\end{array}$

B

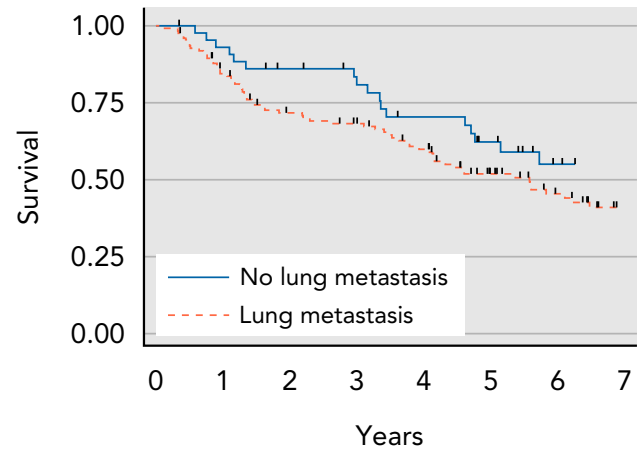

Number at risk

$\begin{array}{lllllllll}\text { No lung metastasis } & 44 & 40 & 35 & 31 & 26 & 20 & 13 & 11\end{array}$

$\begin{array}{lllllllll}\text { Lung metastasis } & 124 & 101 & 82 & 75 & 64 & 45 & 33 & 21\end{array}$

C

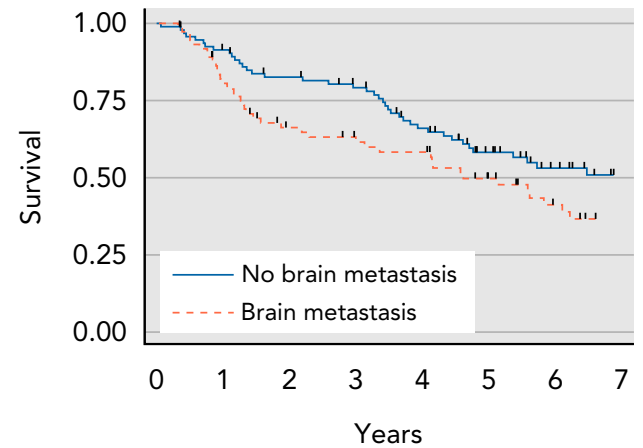

Number at risk

$\begin{array}{lllllllll}\text { No brain metastasis } & 94 & 84 & 74 & 68 & 54 & 39 & 28 & 20\end{array}$

$\begin{array}{lllllllll}\text { Brain metastasis } & 74 & 57 & 43 & 38 & 36 & 26 & 18 & 12\end{array}$

eFigure 1. Survival analysis based on prevalent nonadrenal metastatic sites. Kaplan-Meier curves showing univariate overall survival analysis based on the presence of $(A)$ liver, $(B)$ lung, or (C) brain metastases from the time of $\mathrm{ICl}$ initiation.

Abbreviation: ICl, immune checkpoint inhibitor. 

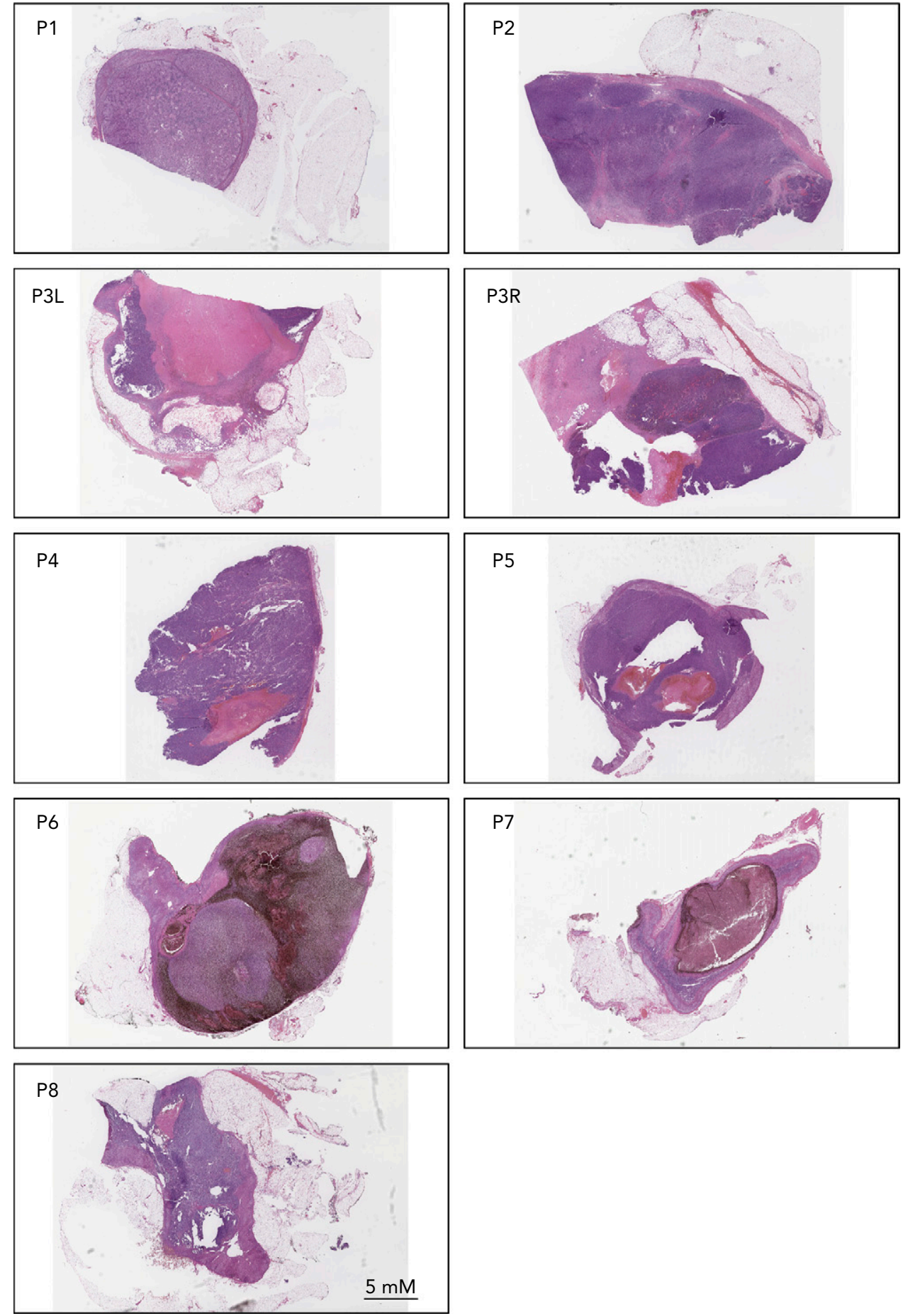

eFigure 2. Immunohistochemical analysis of the AGM microenvironment via H\&E (original magnification $\times 0.5$ ) staining in 9 resected adrenal glands (original magnification: full slide and $\times 4$ ).

Abbreviations: AGM, adrenal gland metastases; $H \& E$, hematoxylin-eosin; L, left AGM; P, patient; $R$, right $A G M$. 
Borgers et al -3
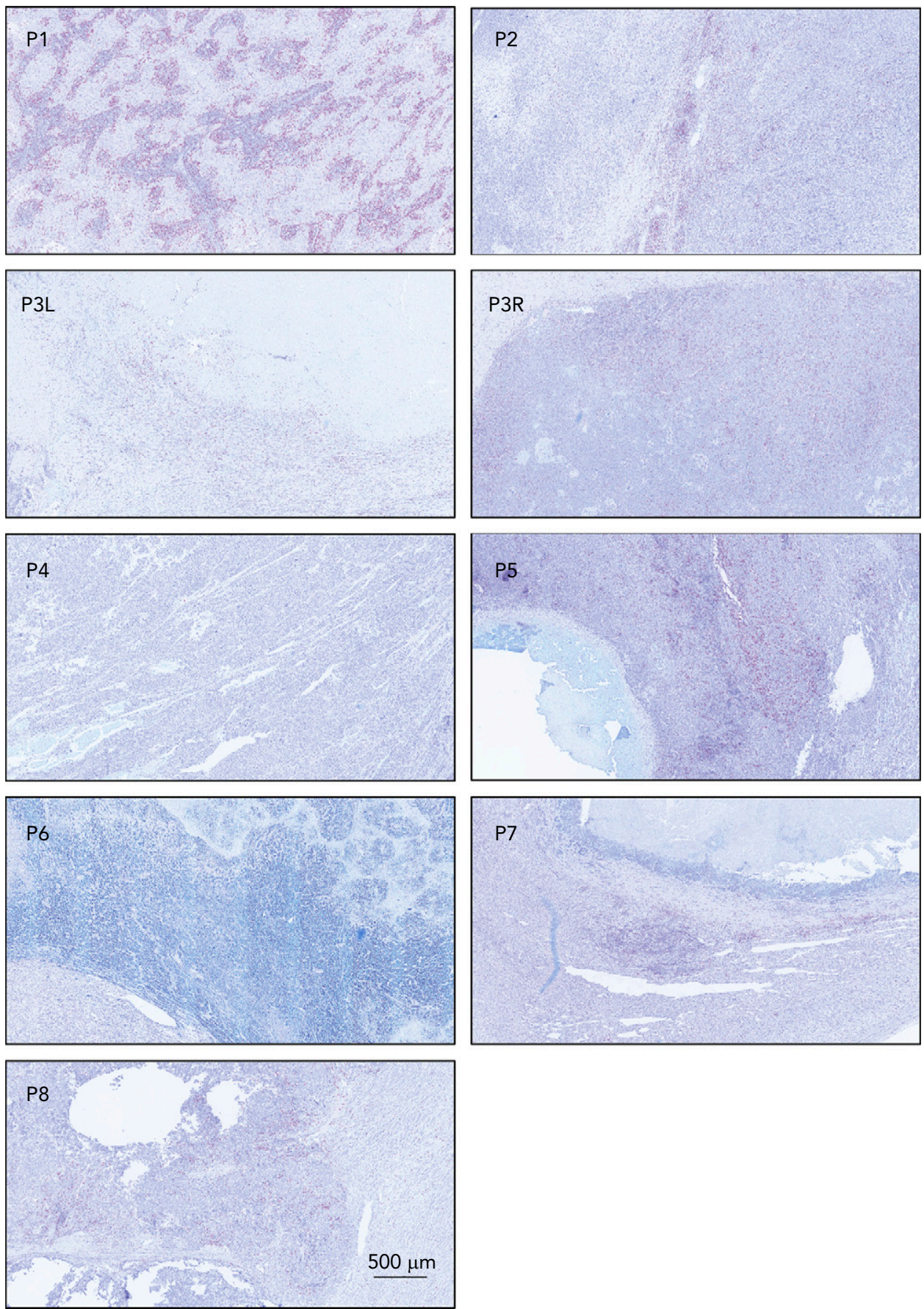

eFigure 3. Immunohistochemical analysis of the AGM microenvironment via CD8 (original magnification $\times 4$ ) staining in 9 resected adrenal glands (original magnification: full slide and $\times 4$ ).

Abbreviations: $A G M$, adrenal gland metastases; $L$, left AGM; $P$, patient; $R$, right $A G M$. 

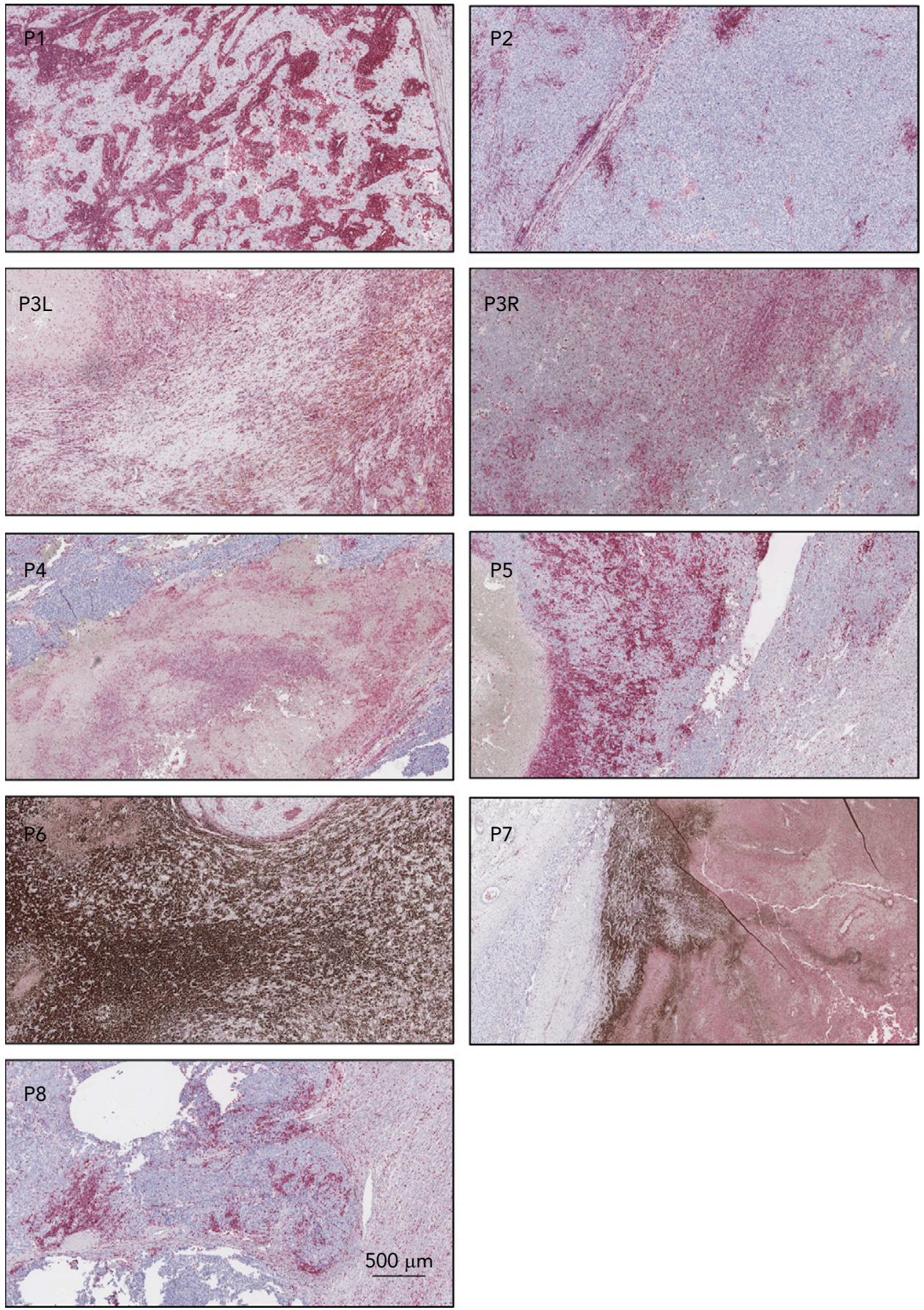

eFigure 4. Immunohistochemical analysis of the AGM microenvironment via CD45 (original magnification $\times 4$ ) staining in 9 resected adrenal glands (original magnification: full slide and $\times 4$ ).

Abbreviations: AGM, adrenal gland metastases; L, left AGM; P, patient; R, right AGM. 